\title{
Real-World Implementation and Outcomes of Health Behavior and Mental Health Assessment
}

\author{
Hector P. Rodriguez, PhD, MPH, Beth A. Glenn, PhD, Tanya T. Olmos, MPH, \\ Alex H. Krist, MD, MPH, Stephanie L. Shimada, PhD, Rodger Kessler, PhD, \\ Suzanne Heurtin-Roberts, PhD, MSW, and Roshan Bastani, PhD
}

Background: Assessing patient-reported health behaviors is a critical first step in prioritizing prevention in primary care. We assessed the feasibility of point-of-care behavioral health assessment in 9 diverse primary care practices, including 4 federally qualified health centers (FQHCs), 4 practice-based research network practices, and a Department of Veterans Affairs practice.

Methods: In this prospective mixed-methods study, practices were asked to integrate a standardized paper-based health behavior and mental health assessment into their workflow for $\geq 50$ patients. We used 3 data sources to examine the implementation process: (1) patient responses to the health assessment, (2) patient feedback surveys about how assessments were used during encounters, and (3) postimplementation interviews.

Results: Most nonurgent patients (71\%) visiting the participating practices during the implementation period completed the health assessment, but reach varied by practice (range, $59 \%$ to $88 \%$ ). Unhealthy diet, sedentary lifestyle, and stress were the most common patient problems, with similar frequencies observed across practices. The median number of "positive screens" per patient was similar among FQHCs (3.7 positives; standard deviation [SD], 1.8), practice-based research network practices (3.8 positives; SD, 1.9), and the Veterans Affairs clinic (4.1 positives; SD, 2.0). Primary care clinicians discussed assessment results with patients about half of the time (54\%), with considerable variation between practices (range, 13\% to 66\%; lowest use among FQHC clinicians). Although clinicians were interested in routinely implementing assessments, many reported not feeling confident of having resources or support to address all patients' behavioral health needs.

Conclusions: Primary care practices will need to revamp their patient-reported data collection processes to integrate routine health behavior assessments. Implementation support will be required if health assessments are to be actively used as part of routine primary care. (J Am Board Fam Med 2014;27:356-366.)

Keywords: Communication, Mental Health, Practice-based Research, Prevention, Screening

Prioritizing prevention within the context of primary care is a key tenet of the Affordable Care Act and is central to the adoption of a patient-centered

This article was externally peer reviewed.

Submitted 30 September 2013; revised 13 December 2013; accepted 19 December 2013.

From the Department of Health Policy and Management, Fielding School of Public Health, University of California, Los Angeles (HPR, BAG, TTO, RB); the Division of Cancer Prevention and Control, Jonsson Comprehensive Cancer Center, Los Angeles, CA (BAG, RB); the Department of Family Medicine and Population Health, Virginia Commonwealth University, Richmond (AHK); The Center for Health Quality, Outcomes and Economic Research, VA Healthcare System, Bedford, MA (SLS); the Department of Family Medicine, University of Vermont, Burlington (RK); and the Division of Cancer medical home model. The development of methods to more accurately assess patient-reported health behaviors in primary care is a critical first

Control and Population Sciences, National Cancer Institute, Bethesda, MD (SH-R).

Current affiliation: Division of Health Policy and Management, University of California, Berkeley, School of Public Health, Berkeley (HPR).

Funding: This research was supported by the National Institutes of Health/National Cancer Institute (grant no. U48DP001946) and the National Institutes of Health/National Center for Advancing Translational Science UCLA Clinical and Translational Science Institute Grant no. UL1TR000124.

Conflict of interest: none declared.

Corresponding author: Hector P. Rodriguez, $\mathrm{PhD}, \mathrm{MPH}$, Division of Health Policy and Management, School of Public Health, University of California, Berkeley, 50 University Hall, Room 245, Berkeley, CA 94720 (E-mail: hrod@berkeley.edu). 
step. Primary care clinicians, however, are faced with many challenges in addressing adult patients' multiple behavioral health issues during traditional 15-minute office encounters. ${ }^{1,2}$ Previous research on the implementation and impact of point-of-care behavioral health assessments has been primarily conducted in practices affiliated with primary care practice-based research networks (PBRNs), ${ }^{3}$ but limited information exists about the implementation of behavioral health assessment in federally qualified health centers (FQHCs) that primarily serve low-income patients.

The National Institutes of Health, in partnership with the Society of Behavioral Medicine, recently led an initiative to identify a brief, practical, standardized set of items to collect patient-reported data on health behaviors, behavioral health, and psychosocial issues that are appropriate for inclusion in an electronic health record (EHR), with the potential to enhance patient-centered care and public health. A 3-phase national expert panel process of consensus building resulted in the identification of core behavioral health measures relevant for primary care: anxiety, depression, stress, sleep quality, smoking, smokeless tobacco use, risky drinking of alcohol, substance use, sugar-sweetened beverage consumption, fruit and vegetable consumption, fast food consumption, physical activity, ${ }^{4}$ and self-rated health. ${ }^{5}$ The expert panel's considerations when selecting these measures included the extent to which evidence-based primary care interventions were available to address the problem health behavior, the value of the information in providing a nuanced understanding of patient health behaviors and clinical data, and their relevance for improving patient-centered outcomes of care.

We conducted a feasibility study to administer an instrument assessing the 13 selected behavioral health measures among nonurgent patients in diverse primary care practices. Each practice implemented the health assessment during a brief intervention period. We assessed the acceptability of the health assessment among diverse patients, examined the extent to which primary care clinicians and/or other team members discussed the assessment results with patients, whether the patients discussed setting goals for improving health behaviors, and whether patients intended to follow-up with their clinician about their concerns. The University of California, Los Angeles, South
General Campus Institutional Review Board (no.12-000297), the Subcommittee on Human Studies of the Veterans Affairs Department of Affairs, and the Virginia Commonwealth University Institutional Review Board (IRB no. HM14523) approved the research study.

\section{Methods \\ Study Setting}

The participating practices were recruited by investigators from 4 research centers located in different geographic areas around the United States. The 4 participating FQHC sites (sites A-D) are in located in the greater Los Angeles, California, region, and each serves low-income patients from different racial and ethnic backgrounds. Site A primarily serves low-income Chinese-American patients, site B primarily serves low-income Mexican-American patients, site C primarily serves low-income FilipinoAmerican and Mexican-American patients, and site D primarily serves low-income Mexican-American and Central-American patients.

Two of the 4 PBRN practices (sites E and F) are located in northeastern Vermont and rural Appalachia Virginia, respectively. The other 2 PBRNaffiliated primary care practice sites (sites $\mathrm{G}$ and $\mathrm{H}$ ) are located in the urban Richmond, Virginia, region. The rural PBRN practices primarily serve white patients and the urban PBRN practices primarily serve African American patients. The participating Veterans Affairs practice (site I) is located in eastern Massachusetts and primarily serves an older, white, lower-middle-class, male Veteran patient population. Table 1 summarizes the primary care practice locations, primary populations served, the practices' history of implementing behavioral health assessments and electronic health record use, length of each practices' intervention period, and the number of health assessment and feedback surveys received.

\section{The Intervention}

In this prospective mixed-methods study, practices were asked to integrate a standardized paper-based health behavior and mental health assessment into their workflow for $\geq 50$ patients. The health assessment was administered by existing primary care staff at the participating practices to 463 adult patients receiving nonurgent care, for example, a return visit or routine/wellness visit, during a 2- to 


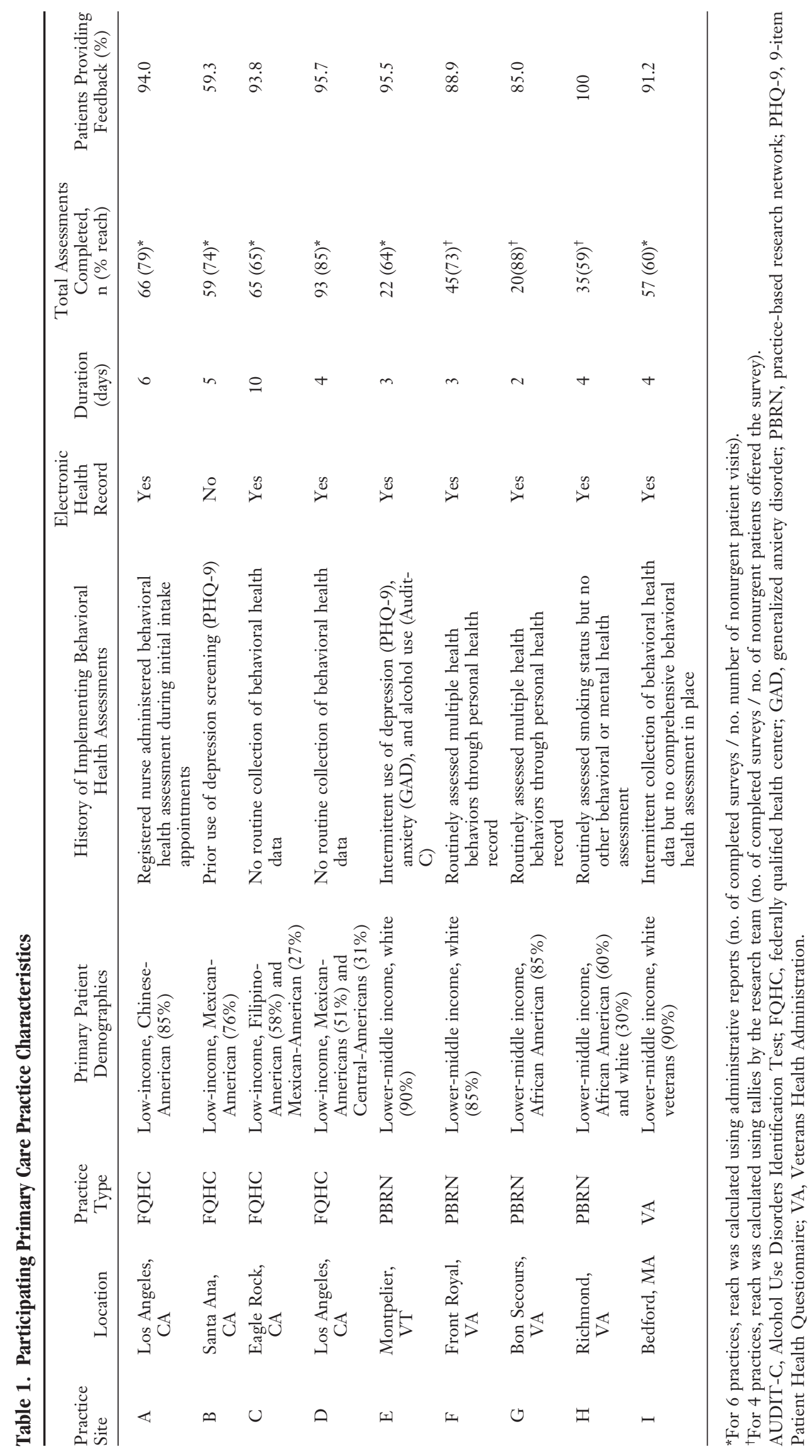


10-day intervention period (during June through September 2012) across the 9 participating practice sites. The assessment was administered in English (sites A-J), Spanish (sites A-D), and Chinese (site A) and was primarily self-administered, although primary care staff helped patients who needed assistance, similar to the practice utilized for other forms completed at the point of registration. The assessment consisted of the 13 brief items assessing health behavior and mental health identified by the national expert panel process as relevant for primary care ${ }^{5}$ and demographic questions. Primary care clinicians and staff were encouraged to discuss the health assessment results with patients during the clinical encounter. Clinicians had access to binders with written guidelines and handouts focused on treatment and referral options for addressing each of the behavioral health and mental health measures covered in the assessment.

\section{Data Collection}

We used 3 primary data sources to examine the implementation and impact of point-of-care behavioral health and mental health assessment: (1) behavioral health assessment responses collected through administration of the instrument to clarify the number and nature of behavioral health issues among patients at each practices, (2) patient feedback surveys to assess how the health assessment was used during their clinical encounter, and (3) interviews with primary care clinicians, staff, and practice stakeholders after the intervention to assess their perspective on the utility of the health assessment in helping patients.

\section{Patient Feedback Surveys}

After each nonurgent encounter, research staff offered patients who completed the health assessment an opportunity to provide their feedback by completing an 8-item survey about the health assessment completion process and the use of the health assessment survey during the clinical encounter, including the extent to which patients felt comfortable completing the questions and whether clinicians discussed the results and goals related to health behavior change with the patient, and whether patients planned to follow up with their provider about health concerns from the health assessment. Participants ( $n=408$; $92 \%$ of health assessment completers) received $\$ 5$ for providing their feedback.

\section{Postintervention Interviews of Primary Care Stakebolders}

We conducted interviews of a convenience sample of primary care clinicians, staff, and clinical leaders at each practice site $(n=20)$ approximately 1 to 2 weeks after the completion of the intervention period in their practice. Interviews assessed the participants' perception of the utility of the health assessment in helping patients with their behavioral health problems at the point of care, barriers and facilitators to implementing the assessment, and their anticipated experiences with using the health assessment or similar assessment as part of routine care in the future. Interviews lasted 30 to $45 \mathrm{~min}$ utes and were recorded and transcribed with the permission of the participants. A \$25 gift card was provided to each participant after the completion of the interview.

\section{Analyses}

First, we calculated the reach of the health assessment. Percentage of "reach" was calculated in 2 different ways because of differences in record keeping, documentation, and workflow among the participating practices. For 6 practices, reach was calculated using administrative reports (number of completed surveys / number of nonurgent patient visits). For 3 practices, reach was calculated using tallies by the research team (number of completed surveys / number of nonurgent patients who were offered the survey). Next, we assessed the extent to which patients' sociodemographic and health status characteristics differed across the 9 practices so that results of the feasibility trial are understood in context. We used $\chi^{2}$ statistics to examine differences for categorical patient variables and $t$ tests to estimate differences for continuous patient variables between the 4 FQHCs, the 5 PBRN practices, and the VA practice.

Using published cut points for "positive screens" or values that would warrant further discussion, ${ }^{4,6-13}$ we calculated the proportion of respondents who would qualify for primary care intervention for each health behavior and mental health measure. We calculated the frequency of each positive screen and the total number of positive screens per patient for each practice and for subgroups of practices (FQHC vs PBRN vs VA). Next, we specified multivariable regression models to clarify the extent to which unadjusted differences in positive screens, based on the primary care practice type, were ex- 
plained by the sociodemographic characteristics and health profile of their patients. We used linear regression to examine the relation of primary care practice type and the total number of positive screens per patient and logistic regression to examine the relation between primary care practice type and screening positive for each of the health behavior and mental health measures. These multivariable models accounted for patient clustering within practices using random practice effects and controlled for patient age, sex, marital status, educational attainment, employment, and US nativity.

We used the patient feedback survey data to examine differences in the use of the health assessment on clinical discussions. To clarify experiences with implementing health assessments at each practice, we analyzed data from interviews with clinicians and staff from the participating practices, which were conducted 1 to 2 weeks following the practice's intervention period. We used a combination of deductive and inductive approaches to analyze the interview data. ${ }^{14}$ We based the initial codebook on the interview guide as well as independent open coding of 4 transcripts by 2 researchers. Coding was compared for consistency, and after consensus was reached, the codebook was revised. Each researcher then coded half the transcripts (or interview notes for unrecorded interviews) using ATLAS.ti software (ATLALS.ti Scientific Software Development GmbH, http://www.atlasti.com/index.html). We analyzed the content of frequently used codes and identified the most consistent themes and patterns of health assessment use across the primary care practice types.

\section{Results \\ Reach of the Health Assessment to Nonurgent Patients}

An estimated $71 \%$ of eligible nonurgent patients visiting during the implementation period returned the health assessment. The reach of the health assessment differed across the participating practices (range, $59 \%$ to $88 \%$ ) (Table 1). The main reasons for not reaching patients as noted by research staff and reported during practice key informant interviews included (1) patients left the practice before the survey could be collected (most common), (2) practice staff forgot to hand out the assessment to some nonurgent patients, especially when clinic staffing was low and/or patient de- mands were high, (3) patients did not want to complete the survey, and (4) the use of researchers to administer the survey in one practice (site I) may have made patients less inclined to participate compared with when primary care team members asked the patients to complete the survey.

\section{Respondent Characteristics}

FQHC respondents were more likely than PBRN or VA patients to be female, have less than a high school education, be nonwhite, be foreign-born; to complete the survey in a language other than English; and to need an interpreter during health care encounters (Table 2).

\section{Positive Screens per Measure}

Fruit and vegetable consumption was the most common "positive screen" for patients because most $(87 \%)$ did not endorse eating $\geq 5$ servings of fruits and vegetables per day. The next most prevalent positive screens were for physical activity (70\%), poor or fair overall self-rated health (54\%), and stress (60\%) (Figure 1). A key finding was that more than a quarter of patients $(26 \%)$ reported recent problem drinking. The most common 7 problem health behaviors were similarly ranked across settings, but a higher proportion of VA patients reported problem alcohol use, anxiety, and high stress compared with FQHC and PBRN patients. FQHC patients were more likely to report fair or poor health status compared with PBRN and VA patients (data not shown).

\section{Total Positive Screens per Respondent}

The median patient had 4 positive screens across the domains, and total positive screens were similar across FQHCs (3.7 positives per patient; standard deviation [SD], 1.8); PBRN practices (3.8 positives per patient; SD, 1.9); and the VA practice (4.1 positives per patient; $\mathrm{SD}, 2.0)$. In multivariate linear regression models accounting for patient sociodemographic characteristics and patient clustering, FQHC patients were more likely to screen positive for low fruit and vegetable consumption (odds ratio [OR], 8.8; $P<.05$ ) and risky alcohol use (OR, 5.0; $P<.05$ ) but less likely to screen positive for fast food consumption (OR, 0.16; $P<.05$ ) compared with PBRN patients (Table 3). In adjusted analyses, VA patients were more likely to screen positive for drug use (OR, 7.34; $P<.05)$ compared with PBRN patients. There were no 
Table 2. Respondent Characteristics by Primary Care Setting

\begin{tabular}{|c|c|c|c|c|c|}
\hline Patient Characteristics & Overall $(\mathrm{n}=463)$ & FQHCs $(\mathrm{n}=284)$ & PBRNs $(\mathrm{n}=122)$ & $\mathrm{VA}(\mathrm{n}=57)$ & $P$ Value \\
\hline Female sex & 61.2 & 70.2 & 65.3 & 3.8 & $<.001$ \\
\hline Age, years & & & & & $<.001$ \\
\hline$<30$ & 5.0 & 5.4 & 4.3 & 3.9 & \\
\hline $30-39$ & 7.66 & 7.6 & 10.3 & 1.9 & \\
\hline $40-49$ & 15.8 & 17.4 & 15.5 & 7.7 & \\
\hline $50-59$ & 32.2 & 37.7 & 24.1 & 21.2 & \\
\hline $60-69$ & 28.4 & 26.5 & 29.3 & 36.5 & \\
\hline $70-79$ & 7.4 & 5.1 & 11.2 & 11.5 & \\
\hline$\geq 80$ & 3.6 & 0.4 & 5.2 & 17.3 & \\
\hline Education & & & & & $<.001$ \\
\hline Less than high school & 34.6 & 50.4 & 11.2 & 3.9 & \\
\hline High school graduate or GED & 25.3 & 21.7 & 33.6 & 25.5 & \\
\hline Some college & 14.8 & 11.0 & 19.0 & 25.5 & \\
\hline Associates degree/technical training & 10.7 & 8.1 & 12.1 & 21.6 & \\
\hline 4-Year college degree or more & 14.6 & 8.8 & 24.1 & 23.5 & \\
\hline Race/ethnicity & & & & & $<.001$ \\
\hline Non-Hispanic white & 29.0 & 6.7 & 69.6 & 82.9 & \\
\hline Black/African American & 7.8 & 1.9 & 23.9 & 9.8 & \\
\hline Mexican-American & 24.8 & 36.3 & 2.2 & 0.0 & \\
\hline Other Hispanic & 13.8 & 19.5 & 2.2 & 2.4 & \\
\hline Chinese & 14.3 & 21.4 & 0.0 & 0.0 & \\
\hline Filipino & 6.8 & 10.1 & 0.0 & 0.0 & \\
\hline Other & 3.8 & 4.1 & 2.2 & 4.9 & \\
\hline Born in the United States & 45.3 & 14.2 & 94.6 & 95.7 & $<.001$ \\
\hline Survey language & & & & & $<.001$ \\
\hline English & 56.4 & 28.9 & 100.0 & 100.0 & \\
\hline Spanish & 31.1 & 50.7 & 0.0 & 0.0 & \\
\hline Chinese & 12.5 & 20.4 & 0.0 & 0.0 & \\
\hline English literacy & & & & & $<.001$ \\
\hline Very good/good & 56.8 & 31.8 & 99.1 & 100.0 & \\
\hline Not good & 20.9 & 33.2 & 0.0 & 0.0 & \\
\hline Not at all & 22.3 & 35.0 & 0.9 & 0.0 & \\
\hline Needs interpreter & & & & & $<.001$ \\
\hline No & 64.7 & 44.8 & 97.4 & 100.0 & \\
\hline Yes & 22.0 & 34.3 & 1.8 & 0.0 & \\
\hline Sometimes & 13.4 & 20.9 & 0.9 & 0.0 & \\
\hline Employment & & & & & $<.01$ \\
\hline Full time & 21.1 & 14.4 & 37.7 & 19.2 & \\
\hline Part time & 12.8 & 18.1 & 3.5 & 5.8 & \\
\hline Unemployed & 16.7 & 21.0 & 9.7 & 9.6 & \\
\hline Homemaker & 15.3 & 23.3 & 3.5 & 0.0 & \\
\hline Disabled & 10.1 & 5.5 & 18.4 & 15.4 & \\
\hline Other & 24.0 & 17.7 & 27.2 & 50.0 & \\
\hline Marital status & & & & & $\mathrm{N} / \mathrm{S}$ \\
\hline Married & 48.5 & 50.0 & 49.6 & 38.5 & \\
\hline Single, never married & 17.3 & 16.9 & 18.3 & 17.3 & \\
\hline Divorced & 12.4 & 10.4 & 10.4 & 26.9 & \\
\hline Other & 21.8 & 22.7 & 21.7 & 17.3 & \\
\hline
\end{tabular}

Data are percentages.

FQHC, federally qualified health center; N/S, no statistically significant differences between primary care practice type; PBRN, practice-based research network; VA, Veterans Health Administration. 
Figure 1. Proportion of respondents screening "positive" for intervention for each health behavior and mental health measure

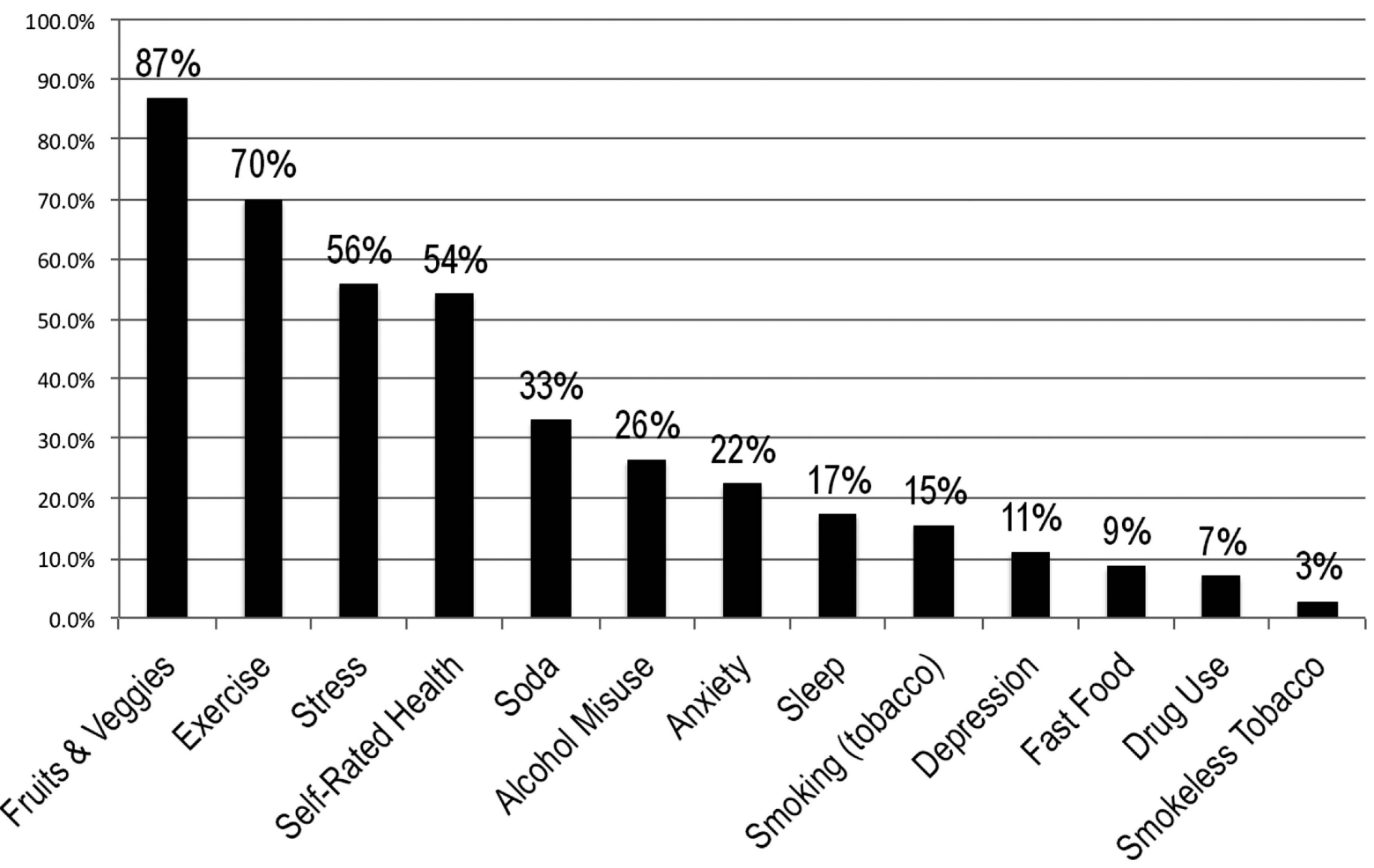

statistically significant differences in the total number of "positive screens" per patient across primary care practice settings (Table 3 ).
The Health Assessment and Clinical Discussions

Primary care clinicians discussed the health assessment results with patients about half $(54 \%)$ the

Table 3. The Relation of Primary Care Practice Type and Screening Positive, Unadjusted vs. Adjusted Analyses

\begin{tabular}{|c|c|c|c|c|c|c|c|c|}
\hline \multirow{2}{*}{$\begin{array}{l}\text { Health Behavior } \\
\text { Measure }\end{array}$} & \multicolumn{4}{|c|}{ FQHC (vs PBRN) } & \multicolumn{4}{|c|}{ VA (vs PBRN) } \\
\hline & Unadjusted OR & $P$ Value & Adjusted OR & $P$ Value & Unadjusted OR & $P$ Value & Adjusted OR & $P$ Value \\
\hline Fast food & 0.22 & $<.001$ & 0.16 & $<.05$ & 0.44 & & 1.06 & \\
\hline Fruits/vegetables & 0.77 & & 8.8 & $<.05$ & 0.93 & & 1.26 & \\
\hline Soda & 0.74 & & 0.76 & & 0.8 & & 1.87 & \\
\hline Exercise & 1.78 & & 1.14 & & 0.89 & & 0.54 & \\
\hline Stress & 1.45 & $<.05$ & 1.25 & & 1.66 & & 2.1 & \\
\hline Anxiety/worry & 0.86 & & 0.65 & & 1.04 & & 1.46 & \\
\hline Depression/interest & 1.06 & & 1.18 & & 1.89 & & 1.14 & \\
\hline Sleep & 0.64 & & 0.82 & & 1.01 & & 1.19 & \\
\hline Smoking & 0.36 & $P<.01$ & 0.52 & & 1.7 & & 0.87 & \\
\hline Smokeless tobacco & 0.31 & $<.05$ & 47.8 & & 1.64 & & 1.75 & \\
\hline Alcohol & 1.1 & & 5.01 & $<.05$ & 1.81 & $<.05$ & 2.47 & \\
\hline Drug use & 1.59 & & 0.68 & & 8.44 & $P<.01$ & 7.34 & $<.05$ \\
\hline Self-rated health & 1.69 & $<.05$ & 1.66 & & 0.77 & & 0.77 & \\
\hline Total positive screens* & -0.05 & & 0.2 & & 0.37 & & 0.6 & \\
\hline
\end{tabular}

$P$ values are compared to practice-based research network (PBRN) practices. The adjusted analyses control for patient sex, age, education, race/ethnicity, nativity, employment status, and marital status. Bold values indicate statistically significant results at $P<.05$. ${ }^{*}$ Coefficient is interpreted as the odds of primary care practice type [federally qualified health center (FQHC) or Veterans Affairs (VA)] patients screening positive for the measure compared to PBRN patients.

OR, odds ratio. 


\begin{tabular}{|c|c|c|c|c|c|}
\hline & $\begin{array}{l}\text { Overall } \\
(\mathrm{n}=408)\end{array}$ & $\begin{array}{l}\text { FQHC } \\
(\mathrm{n}=241)\end{array}$ & $\begin{array}{c}\text { PBRN } \\
(\mathrm{n}=115)\end{array}$ & $\begin{array}{c}\text { VA } \\
(\mathrm{n}=52)\end{array}$ & $\begin{array}{c}P \\
\text { Value* }\end{array}$ \\
\hline Patient felt comfortable answering the questions & 85.6 & 91.7 & 76.3 & 77.5 & $<.001$ \\
\hline Provider showed patients the results of the behavioral health assessment & 58.5 & 53.7 & 67.7 & - & $<.05$ \\
\hline Patients received a copy of results to take home & 36.0 & 28.4 & 50.0 & 41.0 & $<.01$ \\
\hline Provider asked patient about their concerns about the results & 54.1 & 46.7 & 67.0 & 64.3 & $<.01$ \\
\hline Provider asked which health concerns patient would like to work on & 60.2 & 48.7 & 81.3 & 71.4 & $<.001$ \\
\hline $\begin{array}{l}\text { Provider helped identify specific steps patient can take to address } \\
\text { concerns }\end{array}$ & 72.1 & 64.6 & 85.6 & 81.5 & $<.01$ \\
\hline $\begin{array}{l}\text { Patient plans to follow up with provider about health concerns from the } \\
\text { behavioral health assessment }\end{array}$ & 83.2 & 77.5 & 91.1 & 93.2 & $<.01$ \\
\hline
\end{tabular}

Data are percentages.

${ }^{*} P$ values indicate the overall differences across primary care practice types.

FQHC, federally qualified health center; PBRN, practice-based research network; VA, Veterans Health Administration.

time (Table 4), with considerable variation across the 9 practices (range, $13 \%$ to 66\%) (Table 3). Nevertheless, $72 \%$ of patients indicated that their primary care physician (PCP) helped identify specific steps for improving their health. Also, most patients were comfortable answering the health assessment questions $(86 \%)$ and most $(83 \%)$ indicated that they planned to follow up with their provider about concerns from the health assessment.

\section{Perceived Utility of the Assessment}

Duplication of effort often was raised as an issue because the practices' health plans (payers) required their own health assessments, which were often much longer, did not use validated questions, and were considered by participants as less actionable compared with the health assessment implemented as part of the feasibility study. For example, one health plan's intake form included more than 100 questions and patients often need assistance with reading the form content. Clinicians and staff consistently reported that the project's assessment was much shorter, easier to use, and asks more specific and actionable questions compared with the health assessments used by the practice. Several PBRN stakeholders, however, noted that their patients do not have time to complete even brief health assessments. As one PBRN PCP said, "I don't feel like patients really take the time and attention to complete surveys. . . . I feel like it's viewed as more of an aggravation. ... We have more of an urgent care setting lately, or atmosphere, where patients just want to come in and out ... they don't want to be bothered with surveys."

\section{Preferences for Routinely Administering the Health Assessment}

Key informants of PBRN and FQHC practices noted similar preferences for how the health assessment should be administered, that is, before the clinical encounter, self-administered by the patient in their preferred language, and scored and ready to use by the PCP. PBRN and FQHC clinicians and staff differed in their expectations for patient completion. PBRN key informants preferred that patients complete the health assessment before the date of the clinical encounter. For example, the health assessment could be administered through a secure web portal, and the responses could be automatically scored and incorporated into the HER, and the results then could be used during the clinical encounter. The VA and PBRN practices generally do not serve large numbers of non-English-speaking patients, and VA and PBRN key informants expressed hesitance about providing the health assessment in other languages because of the time and resources that would be necessary (eg, online or telephone translation services). As a VA clinician said, "I think it would be just the family member that they brought with them or a telephone translator, but the latter takes time to arrange and there is often no time to do that, even in the regular visits."

FQHC PCPs often identified staff members who could help patients fill out the assessment in 
the patient's preferred language, manually score the assessment, and then provide the results to the physician for use during the clinical encounter. Despite strong preferences for staff support to assist patients as they complete and interpret the assessment results, FQHC stakeholders were often skeptical that routine staff-supported completion would be possible. As one PCP noted, "At safety net institutions . . because of pay rates for medical assistants, it can be challenging. . . . The number of [medical assistants] that we have and their skill level make it challenging to raise expectations.... I think a lot of places like ours, the ability to spread out and reassign those tasks are a little more challenged compared with other practices."

\section{Resources to Address Patients' Behavioral Health Needs}

Key informants across the practices indicated that referring patients to appropriate care to address positive screens for mental health, substance abuse, nutrition, and physical activity promotion were limited by the few referral sources available in the community and the extent to which patients have health insurance coverage that includes these supportive services. Key stakeholders across the practices indicated that health behavior interventions often are "crowded out" by more immediate clinical concerns during PCP-patient encounters. As one PCP noted, “... what ends up happening is just the reality of the situation is that the medical issues are addressed .... and then when it comes to patient health promotion, that part falls to the bottom of the list."

\section{Discussion}

Our feasibility study of routine health behavior assessment in diverse primary care settings demonstrates that practices can implement point-of-care behavioral health assessments and that patients are generally comfortable answering the questions. The reach of the health assessment was good (71\%), but some challenges to reach were observed, including practice staff forgetting to hand out the assessment to some nonurgent patients, especially when clinic staffing was low and/or patient demands were high. Clinicians, however, encountered many challenges in using the health assessment to assist patients during clinical encounters. The mean number of "positive screens" per patient was high (median, 4) across diverse primary care settings, underscoring that routine point-of-care health assessments may increase the number of issues that may need to be addressed during a visit. The nature of behavioral health and mental health problems and the number of problems per patient were similar across diverse primary care settings. Primary care clinicians' use of the behavioral health assessment, however, varied considerably across practices. Although practice interview participants expressed that routine behavioral health assessment would be ideal for patient care, many believed that their practice did not have sufficient internal or referral resources to address all patients' behavioral health and mental health needs. If routine assessment were to be implemented, better decision support for primary care teams, fostering community linkages, and technical assistance will be necessary to aid primary care teams in helping patients prioritize their health behavior improvement goals and monitor their progress. Importantly, efforts now are underway to examine the impact of decision support to aid clinicians and patients in using electronic patient-reported outcomes (PRO) data of health behavior and mental health when discussing health behavior change, setting goals, and monitoring behavior change. ${ }^{15}$

Our study has some important limitations. While it has the advantage of studying the implementation of point-of-care health assessments in diverse practices, all practices volunteered to participate. Even with volunteer practices and a short intervention period (2-10 days), 3 practices faced major challenges in using the health assessment information during clinical encounters with patients. As a result, we believe that our study captured a diverse range of experiences of point-of-care implementation of behavioral health assessments. Most of the participating practices serve low- to lower-middle-income patients that may have more need for behavioral health support. The fact that the number of positive screens did not differ by practice type suggests that most primary care practices are likely to have a significant need for behavioral health support. Also, we were unable to assess the extent to which behavioral health issues would be discussed during clinical encounters without the health assessment. Previous research, however, suggests that clinical discussions about behavioral health and mental health ${ }^{16}$ occur much less frequently than the level of clinician-patient discus- 
sions reported by patients in this study. Finally, a chart audit was not conducted to assess the concordance of self-reports and clinical data. Chart audits, however, have their own limitations because of incomplete and inconsistent documentation of behavioral health discussions.

Facilitating PCP-patient discussions about health behaviors and mental health issues seems to be challenging in some practices because of time constraints; the increasing responsibilities of medical assistants, who need to provide on-site support for patients to complete the assessment; and limited referral resources for mental health, substance abuse, nutrition, and physical activity promotion. If EHR meaningful use requirements extend to collecting, reporting, and using comprehensive behavioral health assessments, ${ }^{17,18}$ primary care practices are likely to face major challenges in using behavioral health data to improve clinical care. As health reform unfolds and primary care practices are expected to integrate routine health behavior assessment, practices will be faced with a need to revamp their patient-reported data collection processes. Technical assistance in the form of training on the use of behavioral health assessments, practice facilitation, ${ }^{19}$ structured rapid-cycle quality improvement support, ${ }^{20}$ and interorganizational learning opportunities $^{21}$ may aid practices in using PRO data and disseminating best practices in supporting patient health behavior change in low-resource settings. Unless implementation support is provided to practices, the routine collection and meaningful use of behavioral health data will likely flounder, particularly in practices with few resources that serve the most vulnerable patient populations. Payment reform and integration of behavioral health measures into EHRs will be important for accelerating the use of PRO data to aid patients in their health behavior change efforts, since duplicative data collection and clinical information systems were noted as barriers to the meaningful use of PRO data.

We thank Julie Volkman; Suzi Spear, PhD; Dylan Roby, PhD; Mark Kelly; and Melissa Hayes for their implementation support and assistance to the research project. We are especially grateful for the steadfast leadership of Russell Glasgow, $\mathrm{PhD}$, and for his oversight of the collaborative process, and critical feedback. Finally, we thank the patients, clinicians, and staff from the 9 practices for their active participation in the project.

\section{References}

1. Fiscella K, Epstein RM. So much to do, so little time: care for the socially disadvantaged and the 15-minute visit. Arch Intern Med 2008;168:1843-52.

2. Bodenheimer T, Laing BY. The teamlet model of primary care. Ann Fam Med 2007;5:457-61.

3. Fernald DH, Dickinson LM, Froshaug DB, et al. Improving multiple health risk behaviors in primary care: lessons from the Prescription for Health Common Measures, Better Outcomes (COMBO) study. J Am Board Fam Med 2012;25:701-11.

4. Coleman KJ, Ngor E, Reynolds K, et al. Initial validation of an exercise "vital sign" in electronic medical records. Med Sci Sports Exerc 2012;44: 2071-6.

5. Estabrooks PA, Boyle M, Emmons KM, et al. Harmonized patient-reported data elements in the electronic health record: supporting meaningful use by primary care action on health behaviors and key psychosocial factors. J Am Med Inform Assoc 2012; 19:575-82.

6. Kroenke K, Spitzer RL, Williams JB, Lowe B. An ultra-brief screening scale for anxiety and depression: the PHQ-4. Psychosomatics 2009;50:613-21.

7. Snowden A, White CA, Christie Z, Murray E, McGowan C, Scott R. The clinical utility of the distress thermometer: a review. Br J Nurs 2011;20: $220-7$.

8. Cella D, Lai JS, Nowinski CJ, et al. Neuro-QOL: brief measures of health-related quality of life for clinical research in neurology. Neurology 2012;78: $1860-7$.

9. Wiener RC. Association of smokeless tobacco use and smoking in adolescents in the United States: an analysis of data from the Youth Risk Behavior Surveillance System survey, 2011. J Am Dent Assoc 2013;144:930-8.

10. Smith PC, Schmidt SM, Allensworth-Davies D, Saitz R. Primary care validation of a single-question alcohol screening test. J Gen Intern Med 2009;24: 783-8.

11. Smith PC, Schmidt SM, Allensworth-Davies D, Saitz R. A single-question screening test for drug use in primary care. Arch Intern Med 2010;170: 1155-60.

12. Paxton AE, Strycker LA, Toobert DJ, Ammerman AS, Glasgow RE. Starting the conversation performance of a brief dietary assessment and intervention tool for health professionals. Am J Prev Med 2011; 40:67-71.

13. Rohrer JE, Herman DC, Merry SP, Naessens JM, Houston MS. Validity of overall self-rated health as an outcome measure in small samples: a pilot study involving a case series. J Eval Clin Pract 2009;15: 366-9.

14. Fereday J, Muir-Cochrane E. Demonstrating rigor using thematic analysis: a hybrid approach of induc- 
tive and deductive coding and theme development. Int J Qual Methods 2006;5:80-92.

15. Krist AH, Glenn BA, Glasgow RE, et al. Designing a valid randomized pragmatic primary care implementation trial: the my own health report (MOHR) project. Implementation science;IS $2013 ; 8: 73$.

16. Makoul G, Dhurandhar A, Goel MS, Scholtens D, Rubin AS. Communication about behavioral health risks: a study of videotaped encounters in 2 internal medicine practices. J Gen Intern Med 2006;21:698703.

17. Glasgow R, Emmons KM. The public health need for patient-reported measures and health behaviors in electronic health records: a policy statement of the Society of Behavioral Medicine. Transl Behav Med 2011;1:108-9.
18. Glasgow RE, Kaplan RM, Ockene JK, Fisher EB, Emmons KM. Patient-reported measures of psychosocial issues and health behavior should be added to electronic health records. Health Aff (Millwood) 2012;31: 497-504.

19. Nutting PA, Crabtree BF, Stewart EE, et al. Effect of facilitation on practice outcomes in the National Demonstration Project model of the patient-centered medical home. Ann Fam Med 2010;8(Suppl 1):S33-44; S92.

20. Rubenstein LV, Chaney EF, Ober S, et al. Using evidence-based quality improvement methods for translating depression collaborative care research into practice. Fam Syst Health 2010;28:91-113.

21. Nembhard IM. All teach, all learn, all improve? The role of interorganizational learning in quality improvement collaboratives. Health Care Manage Rev 2012;37:154-64. 